\title{
TIO BANDS IN COMPOSITE SYSTEMS
}

\author{
A. MILONE, B. BARBUY \\ IAG-USP, CP 9638, CEP 01065-970, São Paulo, Brazil
}

\begin{abstract}
TiO bands at $\lambda 620 \mathrm{~nm}$ are synthesized. The behaviour of these bands as a function of stellar parameters is studied. Application to composite spectra of one galactic bulge globular cluster and to bulges of elliptical galaxies is also carried out. TiO bands may be useful metallicity indicators.
\end{abstract}

\section{Behr viour of TiO Bands}

$\mathrm{TiO}$ bands in the red and near-infrared regions of the early-type galaxies spectra are strong absorption features(e.g. Bica \& Alloin 1986). A detailed study of strong spectral features is an important link between stellar spectroscopy and population synthesis in composite systems.

In order to study the behaviour of $\mathrm{TiO}$ bands in stars as function of metallicity, effective temperature and gravity, we have computed $\mathrm{TiO}$ and total synthetic spectra at $\lambda \lambda 614.5-627.5 \mathrm{~nm}$, in steps of $0.02 \AA$ and with FWHM=0.8 $\AA$ using the code by Barbuy (1989), for a grid of the atmospheric parameters: $\mathrm{T}_{\text {eff }}=$ $4000,4500,5000,5500 \mathrm{~K}, \log \mathrm{g}=0.0,1.0,2.0,3.0,4.0,4.5,5.0$, and $[\mathrm{M} / \mathrm{H}]=$ $-3.0,-2.0,-1.0,0.0,+0.5$ dex; in the same mode employed by Barbuy et al.(1992b) for the study of the $\mathrm{Mg}$ indices. The used model atmospheres are interpolated in the grids of models by Bell et al.(1976) and by B. Gustafsson(p.c.). The atomic data base consists of lines by Moore et al.(1966), whose the oscillator strengths are obtained by fitting the computed lines to the solar spectrum. The molecular data base consist of the line identification: by J.G. Phillips(p.c.) for TiO $\alpha$ $\left(C^{3} \Delta-X^{3} \Delta\right), \gamma\left(A^{3} \Phi-X^{3} \Delta\right)$, and $\gamma$ prime $\left(A^{3} \Pi-X^{3} \Delta\right)$ systems; by Davis \& Phillips (1963) for $C N$ red system $\left(A^{2} \Pi-X^{2} \Sigma\right)$; and by Phillips \& Davis(1968) for $\mathrm{C}_{2}$ Swan system $\left(A^{3} \Pi-X^{3} \Pi\right)$. The molecular oscillator strengths are calculated using the formulae by Kovacs(1969).

We have measured $\mathrm{TiO}$ and total absorptions, $\mathrm{F}(\mathrm{TiO})$ and $\mathrm{F}(6145-6275 \AA$ )respectively, and analyzed its dependence on metallicity, effetive temperature and gravity. The logarithm of $\mathrm{TiO}$ absorption is strongly dependent on the metallicity for $[\mathrm{M} / \mathrm{H}] \geq-1.0$ dex. A similar dependence exist on $\theta_{\text {eff }}$ in giants and dwarfs with $[\mathrm{M} / \mathrm{H}] \geq 0.0$ dex. There is practically no correlation between $\mathrm{F}(\mathrm{TiO})$ and gravity. Similar results are obtained when we have analyzed the relationship between $\log [\mathrm{F}(6145-6275 \AA)]$ and metallicity, temperature and gravity. The linear correlation between $\log [\mathrm{F}(6145-6275 \AA)]$ and $[\mathrm{M} / \mathrm{H}]$ become stronger and extend to all metallicities(see Figure 1).

\section{Synthetic Grid and Composite Spectra}

We have used the information contained in the $\mathrm{BV}$ colour-magnitude diagram of the galactic globular cluster NGC 6553 , whose metallicity is nearly solar: $[\mathrm{M} / \mathrm{H}]=$ -0.2 dex by Barbuy et al.(1992a).

We have computed a grid of synthetic spectra at $\lambda \lambda 614-648 \mathrm{~nm}$ for ten different 
stages of evolution observed in the NGC 6553 for the solar metallicity only. The metodology is the same applied by de Souza et al.(1992).

We have built a composite spectrum, which represent a sum of the individual synthetic spectra according to the relative number of star in each evolutionary stage and the corresponding flux contribution. The resulting composite spectrum was convolved with FWHM from 3.2 until $9.2 \AA$, in order to adjust to the spectra of composite systems.

We have fitted convolved composite spectra to the globular cluster G1 Bica(1988) spectrum, corresponding to metal-rich globular clusters, and the NGC 4936 elliptical galaxy(E0).

Acknowledgements. The calculations and reductions were carried out with the VAX 8530 of the IAG-USP. Financial support by FAPESP is acknowledged.

Barbuy B.:1989, Ap\&SS, 157, 111

\section{References}

Barbuy B., Castro S., Ortolani, S., Bica, E.:1992a, A\&A,259, 607

Barbuy B., Erdelyi-Mendes M., Milone A.:1992b, A\&AS, 93, 235

Bell R.A., Eriksson K., Gustafsson B., Nordlund A.:1976, A\&AS,23, 37

Bica E., Alloin D.:1986, A\&A, 162, 21

Bica, E.:1988, A\&A, 195, 76

Davis S.P., Phillips J.G.:1963, "The Red System of CN Molecule", University of California Press, Berkeley

Kovacs, I.:1969, "Rotational Structure in the Spectra of Diatomic Molecules", Adam Helger Ed. , London

Moore C.E., Minnaert M.G., Houtgast J.: 1966, NBS Monograph $\mathrm{n}^{\circ}$ 61, Washington

Phillips J.G., Davis S.P.:1968, "The Swan System of the $\mathrm{C}_{2}$ Molecule", University of California Press, Berkeley

de Souza R.E., Barbuy B., dos Anjos S.:1992, AJ (submitted)

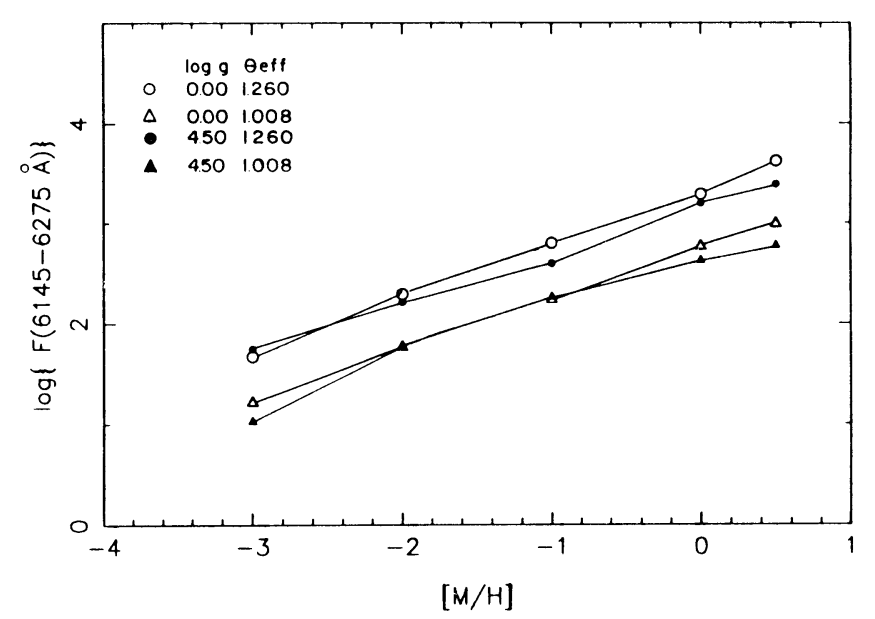

Figure 1 - Logarithm of total absorption as a function of metallicity 